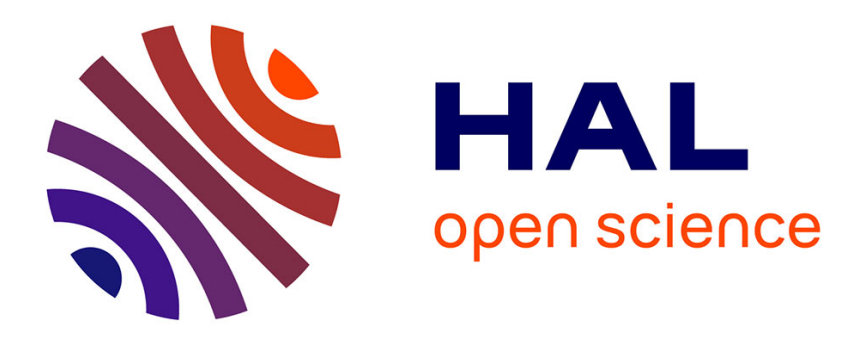

\title{
The indeterminacy bottleneck: Implications for habitable worlds
}

\author{
Ian von Hegner
}

\section{To cite this version:}

Ian von Hegner. The indeterminacy bottleneck: Implications for habitable worlds. 2021. hal03195015

\section{HAL Id: hal-03195015 \\ https://hal.science/hal-03195015}

Preprint submitted on 10 Apr 2021

HAL is a multi-disciplinary open access archive for the deposit and dissemination of scientific research documents, whether they are published or not. The documents may come from teaching and research institutions in France or abroad, or from public or private research centers.
L'archive ouverte pluridisciplinaire HAL, est destinée au dépôt et à la diffusion de documents scientifiques de niveau recherche, publiés ou non, émanant des établissements d'enseignement et de recherche français ou étrangers, des laboratoires publics ou privés. 


\title{
The indeterminacy bottleneck: Implications for habitable worlds
}

\author{
Ian von Hegner \\ Aarhus University
}

\begin{abstract}
It is often assumed that the transition between chemical evolution and biological evolution undergoes an easy and smooth process; that once life has arisen, it will automatically 'flood' a solar system body. However, there is no a priori reason to assume that a link between them is a given. The fact that both chemical evolution and biological evolution meet in a single point, a single cell, can be critical. Thus, one may ask: can a world's environment be favourable for chemical evolution but not for biological evolution, or vice versa? This is an important question worth exploration because certain worlds in the solar system in the past seemed to possess the possibility of chemical evolution, such as Venus and Mars, while several worlds in the present, such as Europa, Enceladus, and even Titan, seem to exhibit such a possibility. Have such solar system bodies thus been, or are, 'flooded' by life? Did they possess the opportunity for biological evolution? The answer depends on the very nature of certain conditions under which evolution occurs, which may indicate that a link between chemical evolution and biological evolution is not automatically realised on a habitable solar system body. Thus, these conditions imply that in the emergence and distribution of cellular life, there exists an indeterminacy bottleneck at which chemical evolution and biological evolution meet through a single cell, whose descendants goes 'information explosive', 'entropy implosive' and 'habitat expansive', which determine whether life moves on to new environments. The consequence is that a world's environment can indeed be favourable for biological evolution, but not for chemical evolution, implying that chemical evolution here does not act as a constraint on biological evolution between solar system bodies. The consequence is also that even if chemical evolution leads to the emergence of a microbial organism in a world, then it is not a given that such a first life form will be subjected to distribution to other environments; and not a given that its existence will continue in the environment it originated in. Thus, the bottleneck may be one of the decisive factors in the differences between habitable worlds and inhabited worlds.
\end{abstract}

Keywords: astrobiology, chemical evolution, biological evolution, exoworlds.

\section{Introduction}

The universe presents us with a myriad of profoundly fascinating phenomena, all worthy of a scientific exploration. Astrobiology is a multidisciplinary field that investigates the deterministic conditions and contingent events with which life arises, distributes, and evolves in the universe, which requires the utilization of several approaches and tools from multiple disciplines. Underlying all the study objectives of astrobiology is ultimately abiogenesis or chemical evolution, that is, the natural assembly of complex molecules into living entities; and biological evolution, which addresses the fact that the universe contains an ability for these entities to use materials in the environment to generate variable copies of themselves, all occurring in mutual competition.

It is often assumed almost implicitly that once life has arisen, it will automatically 'flood' a world. It is believed that the transition between chemical evolution and biological evolution occurs through a smooth and easy process. Indeed, the Earth we see today is virtually covered with life that has colonised almost every available environment on the planet. One may thus assume that as soon as life arises, this is inevitable. If chemical evolution demonstrates sufficient robustness for occurrence and for generation of life on a world like the Earth or elsewhere, even under harsh conditions, then one may automatically assume that biological evolution may also display robustness for such an occurrence and result in the continuation of life as well.

However, this need not necessarily be the case. That an environment can be favourable for the assembly of a cell does not necessarily imply that this or other environments are favourable for the subsequent survival of the cell. It may indicate that we have simply become accustomed to perceiving that such a link exists. While chemical evolution is probably a non-trivial process, triggering and subjecting the first autonomous cell into biological evolution is probably also a non-trivial process. Thus, it may not be a given that individual organisms from the first habitat will proceed into new environments or will proceed to exist in their own habitat. Thus, the transgressions between these two may not be smooth and trivial. 
Thus, one may ask: can a world's environment be favourable for chemical evolution, but not for biological evolution, or vice versa?

A straightforward answer may, of course, be that as soon as the first fully autonomous cell emerges, the cell and its habitat may be subjected to destruction by an incoming meteorite. However, based on the very nature of the conditions under which evolution occurs, the answer may be different and more complex.

This is an important question worth investigation as certain worlds in the solar system in the past seemed to possess the possibility of chemical evolution, such as Venus and Mars, while several worlds in the present, such as Europa, Enceladus, and even Titan, seem to exhibit such a possibility. Have such solar system bodies thus been, or are, 'flooded' by life?

While both chemical evolution and biological evolution are guided by teleomatic processes, that is, processes resulting from the physical laws of nature, it is still the case that the very nature of certain conditions under which evolution operates, for example, the occurrence of contingent events, may indicate that a link between chemical evolution and biological evolution is not automatically realised on a habitable solar system body. Thus, when we explore the conditions of life in the universe, and include the evergrowing number of exoworlds that are being discovered, then the separation and link between chemical evolution and biological evolution can be important to keep in mind.

Thus, the question of whether a world's environment can be favourable for chemical evolution but not for biological evolution, or vice versa, warrants further clarification. This aspect will be discussed in this study.

\section{Discussion}

Information on the indeterminacy bottleneck will be presented in section 3, in which the differences in chemical evolution, explained as a narrowing down of molecules leading to a concentration of structured matter, and biological evolution, explained as an expansion of organisms leading to an increase in structured matter, have been discussed. Next, the reverse is discussed in section 4 , in which a shift between chemical evolution and biological evolution has been described. In sections 5 to 5.5, we have discussed that although it is often implicitly assumed that the transition between chemical evolution and biological evolution occurs easily and smoothly, this is not necessarily the case. Finally, in section 6, we have provided a conclusion of the information presented herein.

\section{The indeterminacy bottleneck}

Bottlenecks are common occurrences in biological evolution and possibly also in chemical evolution. However, at the beginning and at the distribution of life, a crucial and unique bottleneck prime will here be argued to have existed (see Figure 1).

Chemical evolution can be symbolically described as the contents of a conical flask on a solar system body, where the whole body of the flask is a mixture of several different chemical processes distributed over time and place. It begins with multiple simple chemical reactions occurring at the bottom of the conical flask, with several simple chemical products. The contents of the flask with various chemical reactions and products probably did not emerge through a linear process, but might have been generated through processes occurring over substantial parts of the planet and were eventually collected in one area; alternately, they might have been generated constantly in a relatively restricted area. In either case, a conical flask analogy can be used.

Thus, all these processes may have initially begun in or required a considerable volume. However, gradually, it was narrowed down, working upwards in the flask for the occurrence of more complex reactions, to enable more distinct autonomous processes in the last phase to assemble a unit comprising a container (membrane), metabolism, and genetic information, a phase that necessarily occurred in a restricted area. All these processes ended with the generation of an end product, a single autonomous cell that passed through the neck of the conical flask. With this, chemical evolution had reached biological evolution, which could similarly be illustrated as the contents of another conical flask. Thus, although the line between chemical evolution and biological evolution may be unclear, there is a clear line here.

The nature of this first cell, and whether there was such a first cell, is debated. The available evidence suggests that all currently known life forms, which are grouped into the three domains of life, namely 
bacteria, archaea, and eukaryotes, originate from a single universal ancestor, termed as the cenancestor [Fitch and Upper, 1987], which is thus the last common ancestor of all extant life. However, this functional organism, possessing similarities to the modern prokaryote, with complex ribosomes, a coupling between genotype and phenotype [Kim and Caetano-Anollés, 2011], and membranes for chemiosmotic coupling [Gogarten and Taiz 1992], was not the first cell in existence, but merely the one cell among many contemporaries whose lineage succeeded and survived, evolving into all present day life forms, while the lineage of all its contemporaries eventually did not survive.

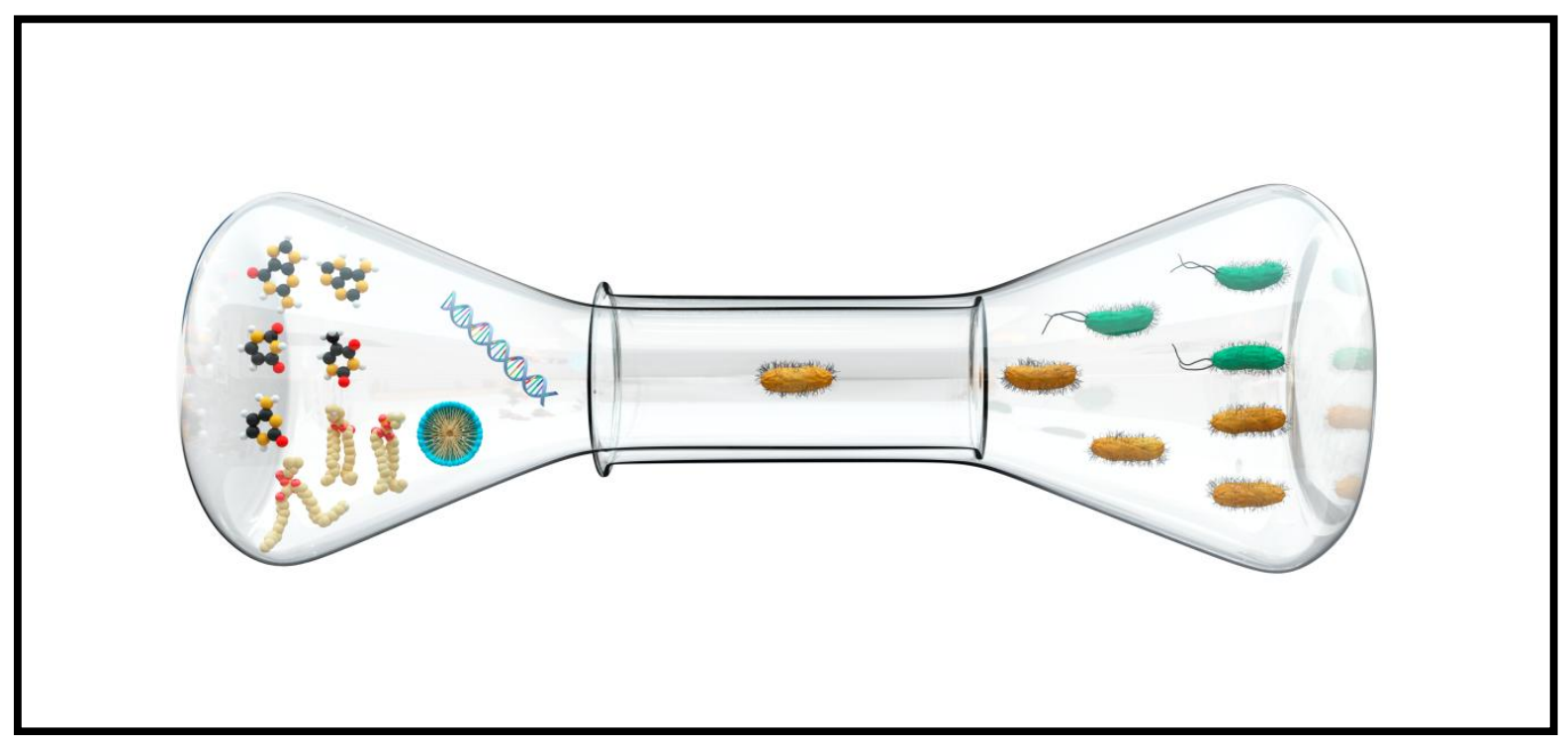

Figure 1. The indeterminacy bottleneck. The body of the left flask shows chemical evolution with a few essential components of the cell, such as nucleobases, phospholipids, a micelle, and a DNA molecule. The body of the right flask shows biological evolution with microbial organisms, with variants in the population occurring as they progress gradually. The dual bottleneck is the juncture where chemical evolution and biological evolution meet through a single cell. The various components and organisms are not depicted in mutually correct proportions.

Thus, the organism that passed through the bottleneck was probably not the cenancestor but existed at an earlier time. Prokaryotic organisation occurs through intermediate steps. An intermediate step in chemical evolution is the generation of self-replicating RNA-like molecules that have been subjected to natural selection already [Rich, 1962; Gilbert, 1986]. Between this RNA world and the cenancestor, it has been assumed that the progenote existed. This is a hypothetical step in the assembly of cells that precedes the prokaryotic cellular organisation [Woese and Fox, 1977]. The progenote may well designate a considerable population of independent pre-prokaryotic cells arising from previous chemical evolution and selection, displaying a few different features observed at the prokaryotic stage, some of which may have eventually merged. Debate and research regarding these stages continue, including the importance of horizontal gene transfer (see e.g. Delaye and Becerra, 2012), which is beyond the scope of this article.

Importantly, between the progenote and the cenancestor, a critical threshold must be eventually achieved for a single cell, and this would confer superiority over previous and co-existing progenote cells; achievement of such a threshold would enable reproduction with its genetic information, metabolic features, and semi-permeable membrane, with all three interacting smoothly through its life cycle and reproduction. This first autonomous rudimentary cell may have emerged shortly before the establishment of a population of cells among which the cenancestor existed, or it may have emerged and established itself long before such a population. What is important is that the cell existed in a certain place and at a certain time.

Thus, all the above mentioned processes ended with the generation of a single autonomous cell. The respective bottlenecks link with each other and meet in a single cell, where chemical evolution narrows down into this one cell, while biological evolution expands outward from this one cell, resembling two triangles that meet each other at their tips. In this sense, they are opposites. They meet at a single point, but exhibit opposite direction in their mechanism. 
In the flask with chemical evolution, there exists an inverse proportionality between the contents at the bottom of the flask and those present near the top of the flask. The processes or products near the bottom of the flask are far less complex than the processes or products near the top of the flask. Thus, the cell that passes through the bottleneck forms the pinnacle of complexity.

However, in biological evolution, the situation is that near the top of the flask, there is a single rudimentary cell with the simplest conceivable functionality. Life begins with minimal complexity as life cannot be simpler than this first functional cell. Near the bottom of the flask, there is a considerable increase in the number of organisms, as well as an increase in cellular complexity. ${ }^{1}$

Thus, if chemical evolution possesses sufficient robustness to generate life on a solar system body, then one may assume that biological evolution also exhibits such robustness for the continuation of life. However, there is no a priori reason to assume the existence of a smooth and easy transition and that a coupling between them is a given. The fact that both chemical evolution and biological evolution meet at a single point, a single cell that is the pinnacle of complexity in chemical evolution, but is merely the simplest conceivable starting point in biological evolution, may be critical indeed.

Chemical evolution, of course, precedes biological evolution; and yet biological evolution poses certain constraints on chemical evolution. While the identity of the environment in which the first cell originated remains debatable, it is evident that regardless of the type of environment in which the last phase of chemical evolution occurred, the cell that emerged underwent necessarily adaptation with respect to this specific environment; otherwise, such an occurrence was not possible. This one specific environment is vital here. The fact that the environment (sub-environment) is favourable to chemical evolution does not imply that it is favourable to biological evolution. Whether the first cell proceeds to biological evolution depends on the conditions of biological evolution, which either allows life's distribution or its isolation.

Thus, while chemical evolution ends at the emergence of the first autonomous cell, biological evolution begins when the first autonomous cell is divided into two cells. In evolutionary biology, life is not determined by considering only a single value, such as a single organism. Instead, it is given by the full variation of the entire system. Thus, biological evolution is always in the plural. Here, it is understood as an expansion. Thus, we obtain a multitude of varying organisms, even within the same initial species that will eventually face a myriad of varying environments. The conditions of biological evolution imply that there is an indeterminacy in whether life moves on to new environments, as discussed in the following sections, and this results in an indeterminacy bottleneck.

\section{Situs inversus}

The questions explored in the introduction were as follows: can a world's environment be favourable for chemical evolution but not for biological evolution? To answer this, one may first ask the reverse question: can a world's environment be favourable for biological evolution but not for chemical evolution?

Chemical evolution, of course, precedes biological evolution in the chain of causality; yet, situations may exist in which biological evolution occur in worlds, at least for a designated duration, when no chemical evolution can occur, because, after all, what does biological evolution require in order to take place?

An environment favourable for chemical evolution is an environment conducive to the formation of complex molecules and their stepwise assembly, while an environment favourable for biological evolution is conducive to the survival and reproduction of organisms.

While evolution is the common denominator between them, there remains a general difference between chemical evolution and biological evolution. To facilitate metabolism, an assembly of matter naturally also occurs in an organism. However, this is guided by teleonomic processes, that is, processes directed by the organism's own DNA. Contingent events that likely play a role in chemical evolution are not present here.

\footnotetext{
${ }^{1}$ An inverse proportionality is not necessarily observed in the flask of biological evolution. Although it is widely believed that single-celled organisms necessarily evolve into multi-cellular organisms, this trend is not observed in the history of life on the Earth; in Darwinian evolution, there are no lower or higher organisms, there is only reproductive success. Thus, microbial life represents the majority of life established in the past and present and will probably continue to represent the majority of life present until the phases that will transform the sun into a red giant end all life forms on this planet.
} 
Thus, chemical evolution is the build up of an autonomous information-bearing unit, while biological evolution is autonomous information-bearing units that act against each other and/or the environment.

Life is, in principle, not trapped on its home world. Thus, it is a well-studied phenomenon that asteroidal or cometary impactors can act as shuttles of matter, acquiring matter from one world and transporting it to the next [Nicholson, 2009]. There is a high probability that matter from the Earth has reached other worlds in the solar system, such as Mars [Beech et al, 2018]. That biomatter has been also transported along with it seems plausible. Therefore, the question is only whether living organisms have arrived as a result of this phenomenon [von Hegner, 2020b]. Thus, lithopanspermia can, in principle, be a vessel for the natural transportation of organisms between solar system bodies or even between solar systems. This poses several related yet distinct situations that are worth discussion.

Mars might have been habitable during its early history, when the atmosphere was probably denser and the planet may have been warm and wet, or cold with melting ice [Fastook and Head, 2015]. It might even be the case that an ocean of liquid water covered the northern Martian plains [Brandenburg, 1987]. However, since the Noachian eon, the possibility of chemical evolution no longer seems to have been present on Mars. Yet the possibility of biological evolution seems to be present in a few locations in the current Amazonian eon on the planet. $^{2}$

Thus, certain terrestrial extremophiles may be able to thrive on Mars, at least for a designated period. An example is Halorubrum lacusprofundi, a microbial organism that is both a halophilic and psychrophilic organism. If this organism through lithopanspermia arrives on Mars from the Earth, and assuming it survives all three stages of lithopanspermia, then it may, in principle, be capable of surviving and reproducing in Martian brines where it can thrive under Martian surface temperatures [Reid et al., 2006].

However, the fact that this environment is favourable for biological evolution does not imply that it is favourable for chemical evolution. Thus, on the one hand, chemical evolution, despite our incomplete understanding of its phases, most likely cannot occur in such brines. On the other hand, Halorubrum lacusprofundi itself did not originate in such brines, and its ancestors have merely gradually adapted from a less extreme environment to this specific terrestrial environment.

Thus, such organisms cannot originate in such brines, and there no longer seems to be a possibility that they could occur elsewhere on Mars under current day conditions. Yet biological evolution has the potential to occur irrespective of the conditions if organisms with such adaptive responses and survival thresholds are placed there. Thus, the important point is that chemical evolution here does not pose as a constraint on biological evolution. The reactions occurring during chemical evolution on one solar system body are irrelevant as soon as the organisms establish on another solar system body. The capacity that organisms have evolved as a result of biological evolution is the only relevant issue that matters here. Thus, in this specific scenario, it is possible for a world's environment to be favourable for biological evolution, but not for chemical evolution.

It has been suggested that life may, in principle, be present between the metallic hydrogen core and the upper atmosphere of Jovian-type planets [Sagan and Salpeter, 1976]. While it is debated whether life can be preserved in the turbulent atmosphere of a gas giant, and all available evidence indicate that no life exists in the atmosphere of the Jovian planets in this solar system, this does not eliminate the possibility that life can indeed exist on some of the many giant exoplanets located around the galaxy.

However, the fact that environments in certain such worlds are favourable for biological evolution does not imply that they are favourable for chemical evolution. Although Jupiter, for example, possesses an environment with numerous ingredients for life, such as water, ammonia, methane, ethane, silicon-based compounds, hydrogen sulphide, oxygen, phosphine, and sulphur [Gibb, 2015], the conditions for chemical evolution do not seem to be present on the planet.

Thus, the primary objects in biological evolution, organisms, are contained within themselves, and new organisms arise from the previously established organisms. However, in chemical evolution, the primary

\footnotetext{
${ }^{2}$ It is possible that life did indeed originate in the Noachian eon and continues to exist in certain locations on the planet; however, this is not relevant to the principal discussion here.
} 
objects are not self-contained; here, a multitude of free complex molecules and sub-processes exist that move separately towards a common assembly, the cell. Despite our incomplete understanding of the phases of chemical evolution, for this multitude of complex molecules and sub-processes to occur, the conditions seem to necessitate the existence of a fluid medium. Thus, chemical evolution may not occur under turbulent gaseous conditions on a gas giant.

However, the conditions for biological evolution seem to be present here. If life arrives through lithopanspermia and survives the turbulence, pressure, temperature, and other conditions in the upper layers of the planet, then it may be possible in this specific scenario for such a world's environment to be favourable for biological evolution but not for chemical evolution.

The previous scenarios countered conditions in which incoming life could evolve in a place where there is no chemical evolution, and we could thus distinguish between chemical evolution and biological evolution as having an asymmetry in relation to each other. A scenario also exists where biological evolution can block the occurrence of chemical evolution. Thus, the incoming life can halt this process, even if chemical evolution in the new world is amidst the process of producing a cell.

As noted already by Darwin (1871), 'It is often said that all the conditions for the first production of a living organism are now present, which could ever have been present ..... at the present day such matter would be instantly devoured or absorbed, which would not have been the case before living creatures were formed'.

The point is that chemical evolution cannot compete against biological evolution. The arriving life will deplete resources that would be required for chemical evolution for the development of a rudimentary cell.

This is also crucial in the case of the Earth. Thus, even though 'the schematics' of chemical evolution are, in a manner of speaking, present on the Earth today, new life does presumably not arise because the various sub-processes leading to life cannot compete with biological evolution. This is not controversial, but it shows that a favourable environment for biological evolution can render the environment unfavourable for chemical evolution. This shows that there is a dichotomy between the two. They do not transgress smoothly into each other.

Of course, these scenarios do not represent a true breach of causality. However, this indicates that although chemical evolution precedes biological evolution on one solar system body, chemical evolution does not act as a constraint on biological evolution between solar system bodies. This asymmetry between chemical evolution and biological evolution is non-trivial because the conditions of biological evolution can also impose constraints on chemical evolution. Thus, one may assume that if chemical evolution can lead to the emergence of a microbial organism in a world, then this life form may continue to thrive and establish itself there as well. However, as will be discussed in the following sections, it is not a given that such a first life form will be able to be distributed to other environments, and also not a given that its existence will persist for a longer duration in the environment in which it originated.

\section{Habitat prime}

As soon as the first autonomous cell emerges, it goes 'information explosive'. Thus, a crucial difference between chemical evolution and biological evolution is that while chemical evolution is likely to be a cumbersome and slow process, biological evolution is fast and exponential.

The first microbial life form that emerged was rudimentary. However, a trait that it shared with its numerous descendants, along with the characteristics of membrane, metabolism, and genetic information, was the ability to divide into two new cells. As soon as the cell achieves this defining feature of division, it goes 'information explosive,' which occurs by binary fission where the number of microbial life forms increases exponentially, which can be expressed as follows:

$$
\mathrm{N}_{\mathrm{n}}=\mathrm{N}_{0} 2^{\mathrm{n}}
$$


where $\mathrm{N}_{\mathrm{n}}$ represents the number of organisms at the end of the time interval, $\mathrm{N}_{0}$ represents the initial number of organisms at the beginning of a time interval, and $\mathrm{n}$ represents the number of generations.

The microbial organism will divide repeatedly, and whatever the favourable environment in which it originated in, will rapidly expand in time and space. This first cell possibly did not possess the ability to move on its own, a feature that is no longer witnessed in many microbial organisms that established subsequently. However, besides being moved around by the potential flow in its environment, this information doubling would be responsible for its movement in the environment, eventually filling it up in the form of a clonal colony, thereby rendering the environment less favourable for themselves, and thus, in a manner of speaking, push themselves out to other environments.

This first microbial organism and its immediate descendants existed in a single habitat; there was only one habitat with life in the entire world. This could be characteristic of the final phase of chemical evolution and is also a critical phase in terms of the commencement of biological evolution.

If we assume that this one environment, this 'habitat prime', where the last phase of cell assembly occurred, had a small cylindrical volume, perhaps the likes of the classic Darwin's warm little pond (1871), how many of these microbial organisms could this pond contain? We assume the pond have dimensions of $0.60 \mathrm{~m}$ in length and $0.15 \mathrm{~m}$ in diameter. Using the volume of the cylinder, we can calculate the volume of the pond at $\mathrm{V}_{1}=\pi \mathrm{r}^{2}$ l. The average size of microbial organisms, such as bacteria, ranges approximately between $\sim 0.4$ and $3 \mu \mathrm{m}^{3}$ [Levin and Angert, 2015]. Thus, we estimate for convenience that the first microbial organism had a length of $2 \mu \mathrm{m}$ and a diameter of $1 \mu \mathrm{m}$. By estimating the volume of this organism using a cylinder, we find that the volume of this organism is $V_{2}=\pi r^{2} l$. Then, we divide the volume of the pond by the volume of this single organism and obtain the number of organisms as expressed below:

$$
\begin{gathered}
\frac{V_{1}}{V_{2}} \\
=6.75 \times 10^{15} \text { organisms. }
\end{gathered}
$$

This number seems to be substantial; however, the increase in a microbial population occurs through geometric progression 1, 2, 4 or $2^{0}, 2^{1}, 2^{2}, 2^{\mathrm{n}}$. The doubling times of unicellular organisms, such as different bacteria, can differ considerably from 9.8 minutes to several hours [Eagon, 1962; Gibson et al., 2018]. Thus, if we apply equation 1 and assume that this microbial organism had a doubling time of 30 minutes, then through exponential growth, it will only require 26.5 hours, comprising 53 generations (if we disregard the time elapsed between each division), to burst the pond with $9 \times 10^{15}$ microbial organisms.

\subsection{Habitats}

The microbial organisms in habitat prime continue to push themselves out of the local favourable environment in which they can exist. They are eventually exposed to a new environment. In the new environment, they will not face competition from other organisms, but they will nevertheless face other environmental stressors. Thus, a model with three different adjacent environments can be established in which the one habitat with organisms can flow (see Figure 2).

A dynamic world environment is never homogeneous. It can be divided into numerous sub-environments which can exhibit considerable variations in the form of temperature, pressure, $\mathrm{pH}$, salinity, and desiccation extremes etc. Thus, a single environment does not exist for biological evolution, but multiple varying environments are observed. This is important because the local environment life originated in obviously possessed the environmental stressors favourable to this life; however, it is not a given that these other environments are favourable to life, or that a specific duration will be available for life to adapt to these other environments.

Thus, the model with three different adjacent environments that can be encountered by the one habitat includes the following: first, an environment that is almost exactly like the habitat the organisms originate in; the next environment poses stressful conditions, but allows the survival of certain individual organisms; and the last environment possesses environmental stressors that are too severe for the survival of organisms. 


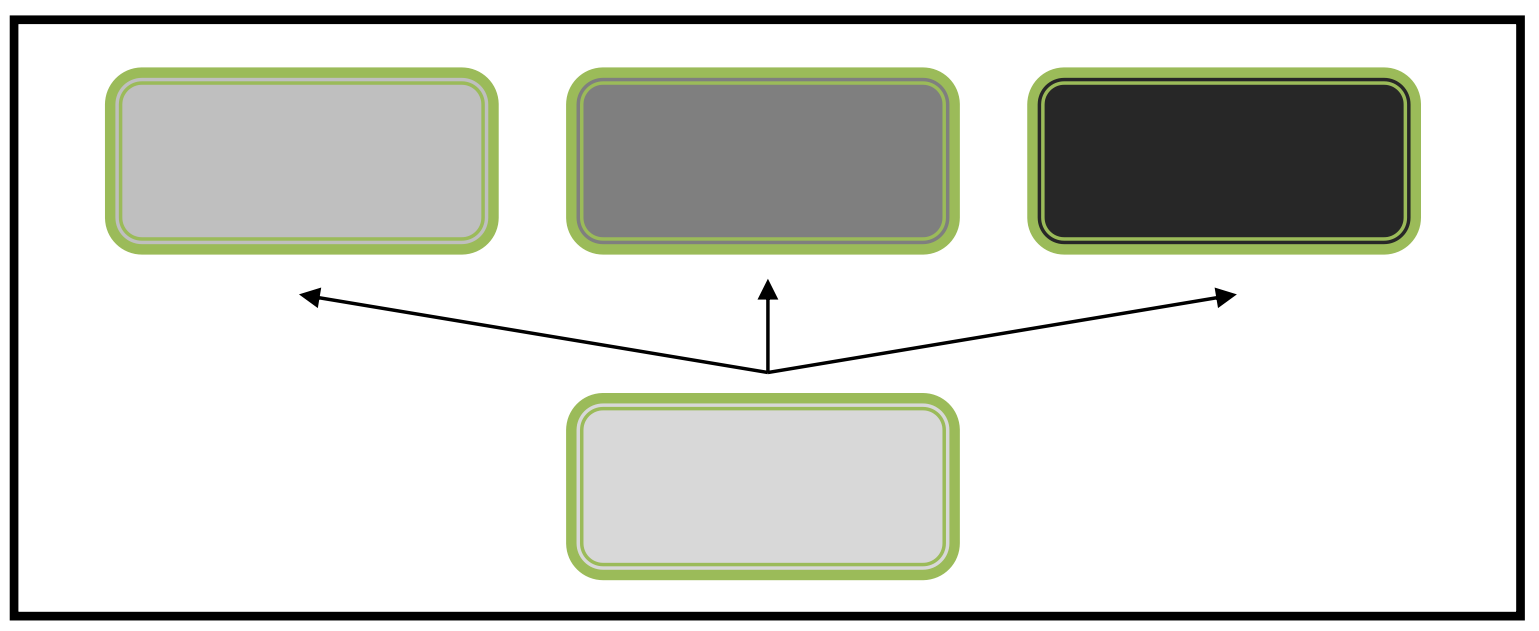

Figure 2. The model with habitat prime and the three different adjacent environments it may encounter. From left to right at the top, an environment that is almost exactly like the habitat the organisms originate in; the next environment poses stressful conditions, but allows the survival of certain individual organisms; and the last environment possesses environmental stressors that are too severe for the survival of organisms.

Therefore, in the model, there is a transition to new favourable or unfavourable environments, which are described by the following expressions:

and

$$
\mathrm{T}_{\text {fav }}:\left(\frac{2}{3}\right)
$$

$$
\mathrm{T}_{\text {unfav }}:\left(\frac{1}{3}\right)
$$

Thus, it can be inferred that the possibility lies in the favour of life gaining a coupling between chemical evolution and biological evolution. However, it is also observed that this coupling is not a given. No smooth or automatic transition occurs between chemical evolution and biological evolution. Thus, here, biological evolution in the form of this 'reverse' bottleneck will pose as a constraint on chemical evolution, almost like a reverse causality.

While chemical evolution pushes the products together to facilitate passage through a single bottleneck, biological evolution begins at a bottleneck, where it spreads outward, rapidly bifurcating into several bottlenecks that life can and must pass through. The environment in which chemical evolution occurred could not necessarily prepare the organisms for encountering the potential adjacent environments where biological evolution must take place because the organisms from this one environment appears with specific adaptive responses and survival thresholds.

This diversity of environments through which microbial organisms must move is one of the reasons why life is not deterministically guaranteed to move on, even if chemical evolution has resulted in the production of a cell. There is a clear contingency sequence involved herein due to the very nature of biological evolution, in which there is an indeterminacy in whether or not life moves to new environments.

This contingency does not usually pose an issue in biological evolution. In fact, environmental diversity is a strength of biological evolution because it acts as a selector of different and more fit variants in a given environment. However, this applies to biological evolution; its place is secured because life is widely distributed. Thus, biological evolution is characterised by a considerable number of different habitats and niches available on a solar system body, all of which represent different environmental stressors. It functions with the availability of a substantial number of variant organisms with different adaptive responses and survival thresholds that have colonised and adapted to a diversity of these environments. If some organisms fail, then others can replace them. If a habitat collapses, then other habitats can replace it. 
However, this secured situation is different from the specific situation discussed here. Environmental flux may not prove to be a strength in the early stages of life's history. Apart from the fact that life forms surviving here will not establish contact with other life forms, but only other environments, there exists only one habitat with life, and there exists only one population of organisms. Thus, life is not presented with an option of another life for continuation if the population disappears. Habitat prime is not presented with the option of any other habitat for salvage if it disintegrates. The various environments existing at the end of the Hadean eon and the beginning of the Archean eon, where terrestrial life originated, were overall more extreme than those witnessed today. Thus, life may not adapt to the environment it encounters in a timely manner owing to the lack of availability of time necessary for adaptation.

In biological evolution, this situation with sharply divided and different environments will usually not occur. Biological evolution occurs across different environments. Thus, among the environments of a world where biological evolution occurs, a range of environments exist at the opposite end of the scale based on factors, such as high temperature, low temperature, high salinity, low salinity, high pressure, and low pressure etc. In two adjacent environments, a clear cut margin/limit is usually not observed. Instead, there is often a gradual transition between the two different environments. Adaptation requires time, and the transition of organisms from a relaxed environment to an extreme environment or vice versa occurs by moving gradually through layers of environments between them, at the marginal areas between them.

However, this secured situation is different from the specific situation discussed here. Life may not adapt to the environment it encounters due to a lack of time. There exists only this one habitat with life, and there is only this one population of organisms. No other habitats exist if disintegration of the present one occurs. No other life exists that may function as a rescue mechanism if the population disappears.

The important thing to realize here is that while it is true that both chemical evolution and biological evolution are guided by teleomatic processes and that biological evolution is also guided by teleonomic processes, contingency events continue to play a decisive role for chemical evolution and biological evolution, as well as for the establishment of a link between them. We cannot ascertain which adjacent environments there will exist or the circumstances under which organisms from habitat prime may encounter them.

\subsection{Habitat intrusions}

As described by expression (3), there is a greater possibility that life will be able to expand into new favourable environments. However, there remains a possibility that it will not expand further, that it will cease expansion if encountering an unfavourable environment, as described by expression (4). If life encounters the last possibility, all microbial organisms will be forced to stay in their environment of origin, where they will be surrounded by a hostile environment. A related but different situation can also be established where the surrounding environment penetrates the boundaries of the safe habitat.

In the previous scenarios, life, even if it encounters a hostile environment that was against its survival, could potentially continue existence in its own native habitat prime. However, it can be imagined that while the organisms live safely in the habitat in which they originate, the boundaries between this and the adjacent environments may break down one day.

The Hadean eon witnessed an extremely violent and changeable time, and the beginning conditions of the Archean eon were also very changeable; hence, this scenario is plausible. If habitat prime is surrounded by e.g. an highly acidic or saline environment, and this unfavourable environment burst in all at once, then it will prove to be fatal for life in habitat prime. Microbial organisms can adapt to highly acidic or saline environments; however, they require time for a step-by-step adaptation, which is not possible here.

The binomial distribution formula can be applied to model this situation in which the probability of obtaining $\mathrm{k}$ successes in $\mathrm{n}$ trials can be calculated. Habitat prime with the first microbial organisms can encounter three different environments outside their own habitat. If each environment is either similar or non-similar to habitat prime, what is the probability of encountering the one environment that is unfavourable to the habitat? We obtained the following numerical values to answer this question:

$\mathrm{n}=3$

$\mathrm{k}=1$ 
$\mathrm{n}-\mathrm{k}=2=$ number of failures

$\mathrm{n}-\mathrm{k}=\mathrm{p}=0.5=$ probability of encountering a similar environment

$1-\mathrm{p}=\mathrm{q}=0.5=$ probability of encountering a non-similar environment

The probability that the population encounters one environment and also does not encounter a specific set of two environments is obtained by the following equation:

$$
\mathrm{P}_{\text {specific } 1 \text { environment }}=(0.5)^{1} \cdot(0.5)^{2}
$$

Furthermore, by applying the binomial coefficient to this equation, the final probability is obtained by the following equation:

$$
\begin{gathered}
\mathrm{P}(\text { match out of } 3 \text { environments })=\left(\begin{array}{l}
3 \\
1
\end{array}\right) \cdot(0.5)^{1} \cdot(0.5)^{2} \\
\approx 0.375 \approx 37.5 \% .
\end{gathered}
$$

Incidentally, the same probability is also applicable if habitat prime encounters one of the other two environments. Thus, the possibility of a coupling between chemical evolution and biological evolution is higher than the opposite trend in regards of an intrusion of an environment into the native habitat. However, this is still not a given.

\subsection{Environmental pressures}

If life encounters the unfavourable situation given by expression (4), all microbial organisms will be forced to remain in their environment of origin, where they will be surrounded by a hostile environment. However, it can be argued that mutations will continue to occur among the population, new variants of organisms with different adaptive responses and survival thresholds will emerge, and natural selection will continue to occur among them. Eventually, they should thus gain the ability to enter the adjacent environment.

However, natural selection will consider the environment in which the organisms live, and not consider the adjacent environment. Thus, organisms that live in e.g. an Antarctic-like environment adapt to the specific pressure, temperature, $\mathrm{pH}$, salinity, and so on, applicable to that environment, and will not adapt to a Sahara-like environment. Natural selection will favour organisms, and thus the population that fits better with the former environment.

However, there is a possibility that variants of organisms that cope with the transition to the latter environment may emerge from time to time because of the occurrence of mutations, although these variants will be few in number. Therefore, we can assume that the following division of organisms exists for the population:

$$
\mathrm{T}_{\mathrm{fav}}:\left(\frac{1}{10}\right)
$$

and

$$
\mathrm{T}_{\text {unfav }}:\left(\frac{9}{10}\right)
$$

This is an extremely generous assumption as there may be billions of organisms, and variants favourable to the new environment may be markedly few. However, this has been done in order to illustrate a point. Thus, this situation is the opposite of the possibilities discussed in expressions (3) and (4).

Thus, if the hostile environment bursts in as explained by expression (6), then there is a possibility that a few individual organisms may survive this catastrophe. However, their presence in the population is a periodic possibility as there will be a substantial selection pressure against these naturally occurring varieties, and they can be rapidly selected out from the population. Therefore, they can only emerge periodically. Therefore, if the adjacent environment penetrates when these individual organisms are not present, the entire population will succumb. 
This would not usually pose an issue in biological evolution because there is a considerable number of habitats and organisms that are available around a solar system body. If some organisms fail, then there are others to replace them. If a habitat collapses, then there are other habitats that can replace it. However, this situation is not applicable here.

Individual variants may also enter the adjacent environment on their own accord. However, these organisms may not exist long enough in their native environment because of the unfavourable selective pressure toward them, to be able to enter the adjacent environment. Their transition to that environment may very well depend on contingency events.

It is also known that settlement in another environment is rarely successful in the first attempt, and several attempts are necessary for settlement through propagule pressure [Lockwood et al., 2005]. This is probably mostly the case for multi-cellular organisms and more rarely for single-celled organisms because of the rapid reproduction and adaptability of the latter.

However, the first population of microbial organisms represents a population of the most rudimentary organism possible. Biological evolution proceeds by resulting in the emergence of several organisms possessing individual variations, and those that experience a selection process to ascertain survival fitness in a new given environment. However, the first cell may have been fragile and less effective than later existing cells. The functions of the first population of organisms may have been too slow or inefficient for the colonisation in other environments compared to the ease of later organisms to do this. Therefore, propagule pressure may be applicable to these organisms. Their possible fragility and poor survival thresholds may be a contributing factor of these individual variants toward the inability of entering the adjacent environment along with the lack of availability of time for adaptation.

However, the possibility exists that individual variants can be fit for one of the adjacent environments and cope with this environment. However, again, the possibility of the opposite trend is greater. Thus, it is not a given that an expansion to new environments will occur.

\subsection{Habitat isolation}

If all microbial organisms are forced to remain in their environment of origin, the pocket where chemical evolution occurred, as described using expression (4), then they will continue to be surrounded by a hostile environment. Therefore, the question is whether life can continue to exist in the restricted habitat in which it originated throughout the rest of its worlds history. As an initial explanation, it may be suggested that the organisms will ultimately continue to survive here for a certain period until they can either leave habitat prime, or until they succumb, which is a binary option described by the expression:

$$
S_{\text {binary }}:\left(\frac{1}{2}\right)
$$

If the microbial organisms remain in habitat prime and continue to divide indefinitely without regard for the establishment of an equilibrium, as described using expressions (1) and (2), then they will inevitably undergo self-destruction. They can burst their habitat and thus allow for entry of the surrounding environment to exert a destructive effect on the only environment in which they can live. They can fill up the expanse of the habitat and thus strangle themselves in the environment in which they emerged, not only leading to an excessive population in the habitat, but also depletion of all available resources faster than they can be renewed. ${ }^{3}$

\footnotetext{
${ }^{3}$ These situations do posses realism. If life e.g. emerged and was established at a hydrothermal vent, then this could have exemplified a habitat with a virtually endless energy source but with limitations in space; the organisms adapt to the parameters applicable close to the source and not to the environmental conditions further away. Thus, the organisms can continue to gain thermal energy but compel each other to move outward as they divide. Those pushed outward to a certain distance will not survive, and the hydrothermal vent where this unique population of life on an entire world exists may eventually cease to exist, as environments are dynamic and continue to change. Thus, organisms must be able to leave this environment to initiate biological evolution, because it is risky to merely stay near such a crack in the ocean floor. Of course, a considerable number of life forms exist today both at hydrothermal vents as well as in their
} 
This occurrence is not observed in microbial populations known today. In biological evolution, a temporary equilibrium will be achieved in terms of organism growth, available resources, energy sources, and space and environmental stressors. When the equilibrium is disrupted, a few species expand their populations further at the expense of other species.

However, it might be hypothesised that these first rudimentary organisms were not subject to this mutual control, and that they, like the RNA molecules in the RNA world, represented replicating machines more than autonomous organisms. Indeed, in principle, life is not always in a self-regulating relationship with the environment.

Thus, it has been suggested that cancer cells behave in their characteristic manner because they regain their original ancient features with unlimited division without regard for the environment and resources [Davies and Lineweaver, 2011]. These cells are incognizant of their necessary requirements. It also seems that if the mammal species Homo sapiens continues its current progression with a growth that will exceed available resources and available space in the only habitat it (thus far) can exist in, then it will potentially destroy the environment before the environment can destroy it (and thus inevitably destroy itself as well).

However, it is more reasonable that the microbial organisms, like their descendants, master the control of this equilibrium. Their downfall would be more attributable to the fact that although life may remain in habitat prime for an extremely long period, environments are dynamic and will change over time. A given environment is subjected to external pressure by adjacent environments, and it is subjected to internal pressure as well because life does not exist passively in an environment, but affects and changes the environment.

Thus, environments are not unshakable fortresses; hence, even if the organisms persistently remain isolated in their habitat, surrounded by hostile environments they cannot adapt to and enter, this habitat, and life, will have to face other environments at a certain juncture. When doing so, the scenarios discussed will be applicable. The microbial organisms may thus sooner or later succumb to the conditions prevailing. Therefore, one can infer that the coupling between chemical evolution and biological evolution is not a given.

On the Earth, life evidently succeeded in undergoing the transition, perhaps even in the first attempt, but it was not a given that it would do so. In a myriad other worlds that seem to possess the possibilities for chemical evolution and biological evolution, it may very well proceed differently. Indeed, if the history of terrestrial life is rolled back to the moment when the first cell emerged and is allowed to be repeated, then it is not certain if life would progress again in the manner it did.

\subsubsection{Isolation}

In principle, it is not possible for organisms to live in a truly isolated environment, and this is not merely due to population growth. As soon as the first autonomous cell emerges, it not only goes 'information explosive,' it also goes 'entropy implosive,' or more precisely, goes 'entropy effective.' It gains the ability to lower its internal entropy at the expense of increasing it in the external environment. Thus, an organism persistently makes its external environment less favourable to itself. This feature is perhaps even more fundamental for life than for reproduction because not all cells can divide, yet live for a certain period, while all cells must inevitably metabolize for survival.

Although the bottleneck does not represent a chemical threshold because chemistry continues through both chemical evolution and biological evolution, the bottleneck represents a turning point in terms of entropy production, or rather, in terms of the efficiency of entropy production.

Any performance of work entails energy conversion and results in an inevitable increase in entropy in the universe. Yet the natural phenomenon designated the cell is markedly distinctive in that respect. Physically, a cell is an open system that exists far from thermodynamic equilibrium. Thus, 'living organisms preserve their internal order by taking from their surroundings free energy, in the form of nutrients or sunlight, and returning to their surroundings an equal amount of energy as heat and entropy' [Lehninger, 1982].

surroundings. However, this holds true for biological evolution that has been secured; life today has secured itself with several habitats to support each other. However, in the past scenario discussed, there existed only one habitat where the organisms could live, and there existed only one population of life. 
The cell is a highly effective entropy enhancer. Thus, similar to natural selection that is a non-random process among organismal variants, the origin of life may be a non-random process as well by which 'the origin and evolution of life... can be understood as resulting from the natural thermodynamic imperative of increasing the entropy production of the Earth in its interaction with its solar environment' [Michaelian, 2011].

The microbial organism is a highly complex chemical system, and a substantial multitude of chemical reactions occur in it. As a clonal colony of microbial organisms should be considered as a biologically immortal entity, in which organisms persistently divide, such a living system is also to be regarded as a persistent entropy-producing system, in contrast to the chemical reactions from which the first cell arose, which eventually ceased on their own.

Thus, an organism automatically makes the external environment less favourable for itself because of the natural thermodynamic tendency of increasing the entropy production of the universe. It can do nothing else to remain alive.

Furthermore, the organism not only increases the entropy of the surrounding environment through metabolism but also do so through maintenance. In principle, chemical evolution may lead to a cell that does not undergo division; indeed, chemical evolution may have resulted in the generation of several non-dividing progenotes before the emergence of a dividing cell. Such a non-dividing cell however cannot continue to exist owing to internal damage and external impacts. The overall maintenance of the cell is established via division into two new cells, whereby through this work, it not only lowers its internal entropy but can also reduce its accumulated internal damage by facilitating it into two new cells.

Thus, organisms must reproduce; they must shift their entropy to the external environment; hence, in principle, it is not possible for them to live in a truly isolated environment.

\subsection{Habitat additions}

If a few microbial organisms move on from their environment of origin, habitat prime, into a new favourable environment as described by expression (3), will life then be secured?

The model with three adjacent environments is applicable to this situation but with a crucial difference. Because now, two adjacent habitats exist, each with its own population of microbial organisms. Thus, life can now go 'habitat expansive.'

In the first scenario, only one way was available. The organisms could move into an adjacent favourable environment or be forced to remain in their environment of origin due to an adjacent hostile environment. However, here the situation is bidirectional rather than unidirectional.

The organisms that have now established themselves in the next favourable environment and have established it as their habitat can once again face three new adjacent environments, namely an environment almost exactly like the habitat in which the organisms live, an environment that offers stressful conditions but allows the survival of certain individual organisms, and an environment with environmental stressors that are too severe for the organisms to survive.

It is assumed that habitat prime is completely surrounded by one of the possible environments, and only one of the adjacent environments can meet at a time; that is, one moves outward like annual rings in a tree trunk. This situation changes after a few rounds, after which diverse environments of different sizes exist. Additionally, organisms in the new environment can also move into the environment from which they emerged, habitat prime. Thus, a new environment is potentially added to the model when a range expansion occurs, as given by the following expression:

$$
\mathrm{T}_{\mathrm{fav}}:\left(\frac{3}{4}\right)
$$

Further, if organisms in the new habitat succeed in entering one of the new adjacent environments, then, a new environment will potentially be added as the range expansion continues as given by the following expression:

$$
\mathrm{T}_{\mathrm{fav}}:\left(\frac{4}{5}\right)
$$


We observe that even when the number of adjacent environments increases, and the number of possible habitats accordingly increases, total access to all adjacent environments is not an option because the possibility is always less than 1 , as there is always, in principle, an environment too severe for life to establish itself into, even when it has sufficient time for adaptation. However, we also note that one here is constantly moving away from the situation described by expression (4), the number of inhabited environments potentially increases, and the sheer number of inhabited environments with a diversity of life increases life's ability to remain in the world.

However, it is not a given, it never is. First, there is no guarantee that the expansion will be greater than the two or three existing habitats. They may be forced to stay in their habitats and may be forced to undergo the situation discussed in section 5.4. Second, the possibility of life remaining on a solar system body would be greater if the first inhabited habitats were located far apart. This occurred at the end of the Hadean eon, where dynamic restructuring of environments was prevalent and the situation discussed in section 5.2. might occur. Furthermore, meteor impactors were prevalent phenomena. Today, a local meteor will not pose a threat to life per se as life has secured itself with redundancies. However, prior to the presently prevailing conditions, it could be a crucial factor. Thus, the possibility of life remaining on one world would be greater if the inhabited habitats were located far apart such that a possible catastrophe in one place could not destroy the other.

In this initial phase, the first habitats were necessarily in close proximity to each other. However, the environments described by expression (11) gradually move away from habitat prime over time and place. Here, despite these initial potential obstacles, the situation will gradually become a familiar scenario in biological evolution. With two inhabited habitats as a minimum, it is now indisputably biological evolution that is at play. The bottleneck prime has been left behind, the role of chemical evolution has been played out, and life proceeds according to the well-known conditions of biological evolution. Life secures itself in the form of a redundancy of habitats and populations it can generally rely on for salvage, and its possibility to hold on is now greater than before, and may continue to increase.

However, one last consideration is applicable in this situation. For both 'information explosive' and 'entropy implosive', the physical processes have characteristically asserted themselves through the active cell. However, with 'habitat expansive', the environmental factors exert more prevalent on the cell, rather than through it, at least in initial stages. With 'information explosive', life can potentially make the external environment less favourable to itself, and with 'entropy implosive' it always makes the environment less favourable. However, with 'habitat expansive', we encounter an initially constrained situation.

In biological evolution, life does not simply exist passively in an environment; life can influence and transform the environment, thus making it more favourable to itself. However, in the early stages of life's history, conditions seemed to be different. Life emerged as a result of chemical evolution and its survival thresholds were constrained by the unique state of its original form; a diversity of life did not exist. It was forced to counter pressure, temperature, $\mathrm{pH}$, salinity, desiccation extremes, and so on, in the adjacent environments head on; there was not enough time for adoption of selective choices and for adaptation. It is only later that life had the opportunity to potentially change its environment and make it more favourable for itself.

Thus, once again, in the early stages, it was not a given that life would continue, as there were constraints on even this possibility. However, life on the Earth was evidently successful in going 'habitat expansive'.

\section{Conclusion}

Underlying all the study objectives of astrobiology are ultimately chemical evolution and biological evolution, and the deterministic conditions and contingency events with which they arise and occur. The bottleneck represents the complexity that characterises both chemical evolution and biological evolution. It may be one of the decisive factors in the differences between habitable worlds and inhabited worlds. It represents a threshold in the form of biological bifurcations or natural selective crossing points that act as pressures or constraints on the further course of life.

Therefore, even if chemical evolution is ultimately a natural consequence of solar system formation, it is not a clear cut given that a link will be established between chemical evolution and biological evolution 
because even though chemical evolution precedes biological evolution, biological evolution continues to pose certain restrictions on chemical evolution.

In its ultimate consequence, this implies that even in a hypothetical scenario with two Earth-like planets, both with virtually the same history and resources and both with rich potential for chemical evolution, it can be predicted that biological evolution occurs on only one world. Conversely, it can be predicted that worlds with environments that are distinct from Earth-like planets, with little potential for chemical evolution, may still possess the conditions for biological evolution.

An environment may be favourable for chemical evolution but unfavourable for biological evolution and vice versa because of the very nature of the conditions under which evolution occurs. Thus, chemical evolution can have, or may currently, occur on solar system bodies such as Venus, Mars, Europa, Enceladus, and even Titan. However, it is not a given that there has been, or could be, biological evolution there. Interestingly, if life is discovered there, then it is not a given that chemical evolution has occurred there: life may have been transported there.

Certain objections may arise. Extremophiles have achieved a preeminence within astrobiological research because of their ability to exist in environments, which, until their existence was reported, was considered too extreme for life. Thus, their use as analogues to potential extra-terrestrial life in our solar system, as well as in other solar systems, has been widely discussed [von Hegner, 2020a].

There is evidence to suggest that thermophiles are among the oldest life forms existing on this planet [Gaucher et al., 2010]. Therefore, it may be stated that if the first life form was an extremophile, then the situation of encountering different adjacent environments should not pose an issue. However, even if it were the case that they belonged to the first group of entities of life emerging on the planet, it does not change the situation that the first life encountered diverse environments, and must possess the ability to cope with one of these environments to establish life. It is important to realise that an extremophile is robust only in its own local environment. An extremophile, which is removed from its local environment, rapidly perishes in another environment: life adapts to local environments, which is an insight of Darwinian evolution.

Thus, if for example, an extreme halophile, an organism whose main characteristic feature is a high salinity requirement for growth and metabolism, is removed from its hypersaline environment and placed in an environment with pure water, then, as everything inside its cell require a high concentration of salts for maintenance, it will actually burst open due to the osmotic pressure of the environment. It is trapped by its local hypersaline environment and can never be instantly displaced from that environment, but requires a gradual adaptation for escape and subsequent survival.

Thus, although extremophiles can live in certain environmental extremes, they are not more robust than mesophiles. Similar to the specific mesophiles that can only exist in specific environments, specific extremophiles can only exist in specific environments. Thus, extremophiles exhibit poor survival with regard to the changing environment. Therefore, even if the first population of organisms was extremophiles, they would not have survived better than mesophiles in terms of coping with one of the three adjacent environments.

Another objection may be based on the key word here, namely a 'single' cell that passed through the bottleneck. Thus, it is possible that chemical evolution produced several independent origins of life almost simultaneously, depending on whether the origin of life represents an improbable or probable event. Thus, there could have been several cellular end products existing almost simultaneously in chemical evolution, similar to the undeniably numerous cells existing simultaneously in biological evolution.

However, even though there could have been a myriad such autonomous cells, biological evolution acted here as a constraint, as available evidence, as mentioned in section 3, indicates that all contemporary terrestrial life forms originate from a single common cell, known as the cenancestor [Fitch and Upper, 1987] or the Last Universal Cellular Ancestor [Philippe and Forterre, 1999], although this was not the first cell in existence. This implies that if there were several origins of life, only one of these succeeded and survived, evolving into the three domains of life, namely bacteria, archaea, and eukaryotes. Biological evolution allowed, in a manner of speaking, only this one cell to pass through and generate descendants in the long run. 
However, this may indeed be different in other worlds in the galaxy and beyond. There may indeed even be life with another independent origin on this planet that is simply not discovered thus far. However, it does not negate the mechanisms discussed in this study.

It may also be argued that the evolutionary strategy, called bet hedging, may facilitate the transition of organisms to a new environment. This strategy enables organisms to cope with more than one environment at a time, even with the unpredictable ones [Seger and Brockmann, 1987]. While this is a valid point, and bet hedging may be an ancient strategy, it remains as a strategy that emerges through evolvability; thus, the first organisms probably did not possess this trait, and it is a strategy that requires time to achieve. However, it may have helped organisms to finally escape their habitat after a certain period.

Other objections may be based on the fact that there remains much information to be revealed regarding chemical evolution. Chemical reactions can obviously occur in different environments, but can chemical evolution occur as well? Biological evolution can occur in different environments, from the most relaxed to the most extreme. However, can chemical evolution also exhibit such an occurrence? Does it require a specific type of environment for the generation of a cell, or is it possible for chemical evolution to occur in two completely different environments and still result in the production of different cells in that environment? Thus, it may be suggested that if a cell can arise in different environments, then the situation of encountering different adjacent environments should not pose an issue.

However, regardless of whether chemical evolution occurs each time in an environment with certain parameters or whether it can occur in environments with other parameters, the generation of each cell will still occur in a certain environment. Furthermore, although different phases of chemical evolution may not occur in the same type of environment, the last phase in which the cell assembles must necessarily occur in a particular environment, and this environment is distinct from other environments. Although two chemical evolutions have resulted in the generation of a cell in different types of environments, each cell is adapted to this specific environment in which it originated, and thus biological evolution will enter the scene, as each cell would encounter different environments in biological evolution, regardless of the particular environment from which it originates.

Thus, a particular cell emerges in a specific environment, and a different cell emerges in another specific environment. However, even though these environments are different from each other, these cells are still on equal footing in that they meet a diversity of environments different from their own; thus, the scenario is the same as that discussed in this article. Therefore, chemical evolution is not conducive to biological evolution per se. Whether the first cell proceeds to biological evolution depends on the conditions of biological evolution itself, not the conditions for chemical evolution.

It is also possible that the bacteria or archaea-like cell represent a blueprint for life which states that life can only arise in this simplest functional form because it is difficult to imagine life in a simpler form than the most rudimentary bacteria or archaea. However, even if chemical evolution occurring on several worlds deterministically results in the generation of the same initial similar life form, and thus poses a restriction for biological evolution by locking the bottleneck to commence at this specific juncture, it is nevertheless, seemingly paradoxical, not certain that these life forms will continue to exist anyway, since biological evolution, which itself follows predictable patterns, in some situations, as discussed, will not allow the continuation of life forms.

Could it not also be stated that some of the different intermediate stages that occurred before the emergence of the first autonomous cell would also encounter different adjacent environments that these stages must be able to enter into? After all, would this not occur in, for example, the RNA world? Here, natural selection, continuation of information, work, and expansion etc. took place. Thus, did RNA molecules not go 'information explosive' as well?

Indeed, there might have been bottlenecks for these processes as well, which might have made the incidence of the emergence and distribution of life in a world discussed thus far even less a given. However, these aspects would constitute chemical evolution occurring in a world; it is not biological evolution that occurs. This occurs only when a reproducing autonomous cell exists. Thus, the key word here is again a 
single cell. Life probably originated from an RNA world; however, all current cellular life forms originated from the cenancestor, which smoothly displayed an interaction between a membrane, metabolism, and genetic information, and this originated from a cell that could smoothly reproduce and pass on these traits. Even viruses that have been subject to arguments for categorisation as an independent domain [von Hegner, 2020c], with some ancient viruses emerging before the first cell discussed herein, depend on organisms from one of the three domains for their continued existence.

Thus, the RNA world, along with the processes that led to membrane and metabolism, ultimately passed through the bottleneck in the form of a single rudimentary but functional cell. If this had not occurred, then perhaps even today we would have had different sub-processes of chemical evolution passing through bottlenecks, and cellular life would not be established anywhere. This is the reason why biological evolution is distinct from chemical evolution.

Even though chemical evolution and biological evolution are both guided by teleomatic processes, and biological evolution is guided by teleonomic processes, contingency events continue to play a decisive role in the distribution of life in a world. Whether the microbial organisms overpopulate and burst their habitat, the surrounding environments burst theirs, or a more peaceful movement occurs, the organisms will eventually establish contact with the surrounding environment. Thus, a sub-environment may be favourable for the final stages of chemical evolution, but the surrounding environments that satisfy the conditions for biological evolution need not be favourable for the distribution of this life. This may eventually imply that this one habitat with this one population of life will disappear.

Thus, there is an indeterminacy involved in the joint bottleneck between chemical evolution and biological evolution that has implications for life's existence and distribution, even in otherwise habitable worlds.

\section{References}

Beech, Martin, Coulson, Ian M. Comte, Mark: Lithopanspermia - The Terrestrial Input During the Past 550 Million Years, American Journal of Astronomy and Astrophysics 2018; 6(3): 81-90.

Brandenburg, John E. (1987). "The Paleo-Ocean of Mars". MECA Symposium on Mars: Evolution of its Climate and Atmosphere. Lunar and Planetary Institute.

Darwin, Charles, Letter to Joseph Dalton Hooker, 1871, in Darwin, Francis (ed) (1887), The life and letters of Charles Darwin, including an autobiographical chapter, 3 vols. John Murray, London.

Davies P.C.W. and Lineweaver, C. H. Cancer tumors as Metazoa 1.0: Tapping genes of ancient ancestors, Phys Biol. 2011 Feb; 8(1): 015001.

Delaye, Luis, Becerra, Arturo: Cenancestor, the Last Universal Common Ancestor, 2012, Evolution: Education and Outreach 5(3).

Eagon,R. G. Psedomonas natrigens, a marine bacterium with a generation time of less than 10 minutes, $\mathrm{J}$ Bacteriol. 1962 Apr; 83(4): 736-737.

Fastook, J.L. and Head, J.W. (2015) Glaciation in the late Noachian icy highlands: ice accumulation, distribution, flow rates, basal melting and top-down melting rates and patterns. Planet Space Sci 106:82-98.

Fitch, W. M., Upper, K. (1987) The Phylogeny of tRNA Sequences Provides Evidence for Ambiguity Reduction in the Origin of the Genetic Code, Cold Spring Harbor Symp. Quant. Biol. 52, 759-767.

Gibb, Bruce C. The organic Solar System, Nature Chem. 7, (2015).

Gibson, Beth, Wilson, Daniel J., Feil, Edward and Eyre-Walker, Adam: The distribution of bacterial doubling times in the wild, Proc Biol Sci. 2018 Jun 13; 285(1880): 20180789.

Gilbert, Walter (1986). "The RNA World". Nature. 319 (6055): 618.

Gogarten, J. P., Taiz, L. 1992. Evolution of proton pumping ATPases: Rooting the tree of life. Photosynthesis Research 33: 137-146.

Kim, K. M., Caetano-Anollés, G. 2011. The proteomic complexity and rise of the primordial ancestor of diversified life. BMC evolutionary biology 11: 140.

Lehninger, Albert (1982). Principles of Biochemistry, Worth Publishers.

Gaucher, E.A.J.T., Kratzer, J.T., and Randall, R.N. (2010) Deep phylogeny-how a tree can help characterize early life on Earth. Cold Spring Harb Perspect Biol 2. 
Levin, Petra A. and Angert, Esther R. Small but Mighty: Cell Size and Bacteria, Cold Spring Harb Perspect Biol. 2015 Jul; 7(7): a019216.

Lockwood JL, Cassey P, Blackburn T (2005) The role of propagule pressure in explaining species invasions. Trends in Ecology and Evolution, 20, 5, 223-228.

Michaelian, K. Thermodynamic dissipation theory for the origin of life Earth Syst. Dynam., 2, 37-51, 2011.

Nicholson, WL: Ancient micronauts: interplanetary transport of microbes by cosmic impacts. Trends Microbiol, 2009 Jun;17(6):243-50.

Philippe H, Forterre P: The rooting of the universal tree is not reliable. J Mol Evol 1999, 49:509-523.

Reid, I.N. Sparks, W.B. Lubow, S. McGrath, M. Lifeio1, M. Valenti, J. Sowers, K.R. Shukla, H.D. MacAuley, S. Miller, T. Suvanasuthi, R. Belas, R. Colman, A. Robb, F.T. DasSarma, P. Müller, J.A. Coker, J.A. Cavicchioli, R. Chen, F. and DasSarma, S, (2006) Terrestrial models for extraterrestrial life: methanogens and halophiles at martian temperatures. Int. J. Astrobiol. 5, 89-97.

Rich A. On the problems of evolution and biochemical information transfer. In: Kasha M., Pullman B., editors. Horizons in Biochemistry. Academic Press; New York, NY, USA: 1962. PP. 103-126.

Sagan, C., Salpeter, E. E.: Particles, environments, and possible ecologies in the Jovian atmosphere, The Astrophysical Journal Supplement Series, 1976.

Seger, J. and Brockmann, H. J. (1987). What is bet-hedging? Oxford Surveys in Evolutionary Biology 4, $182-211$.

von Hegner, Ian: Extremophiles: a special or general case in the search for extra-terrestrial life, Extremophiles (2020a) 24:167-175; Published online: 2019.

von Hegner, Ian: Interplanetary transmissions of life in an evolutionary context, International Journal of Astrobiology, 19, 2020b.

von Hegner, Ian: First principles of terrestrial life: exemplars for potential extra-terrestrial biology, HAL archives-ouvertes.fr| CCSD, 2020c.

Woese, C.R., Fox, G.E. The concept of cellular evolution. J Mol Evol 10, 1-6 (1977). 